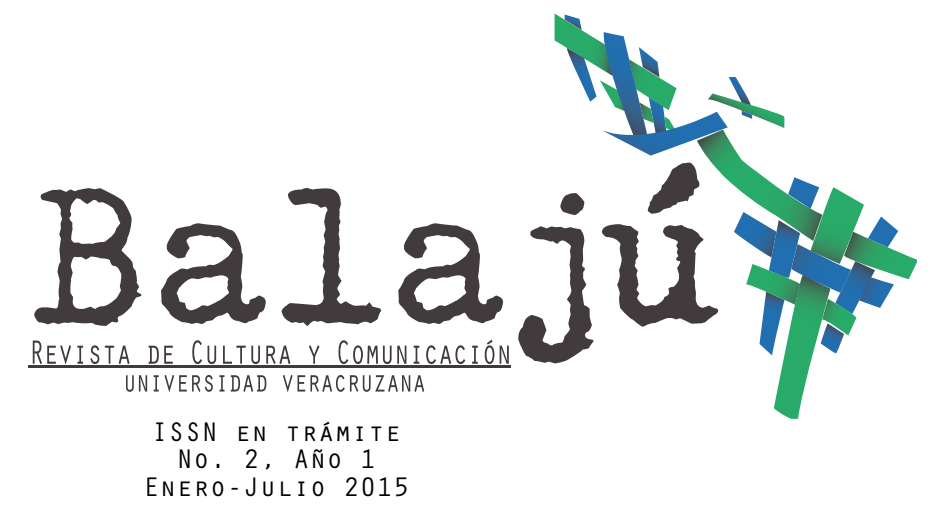

\title{
Música en la guerra contra el terrorismo: el soundtrack de la tortura estadounidense
}

Jorge Loeza*

Universidad Veracruzana, México

joloeza@gmail.com

\section{RESUMEN}

La política de seguridad de Estados Unidos redobló esfuerzos a raíz de los atentados del 11-S, entre estos, la apertura de bases ilegales externas para el tratamiento de detenidos acusados de terrorismo. En estos sitios se evidenciaron prácticas de tortura, incluido el uso de canciones bajo los denominados no-touch methods.

\begin{abstract}
ABSRACT
The security policy of the United States redoubled efforts following the attacks of 11-S, among these, the opening of external unlawful basis for the treatment of detainees accused of terrorism. At these sites, torture practices including the use of songs under the so-called no-touch methods were demonstrated.
\end{abstract}

\section{PALABRAS CLAVES}

Música, Guerra contra Terrorismo, Tortura Sonora

\section{KEYWORDS}

Music, War on Terror, Acoustic Torture 


\title{
Música en la guerra contra el terrorismo: el soundtrack de la tortura estadounidense
}

\author{
"El ejército de Josué utilizó sus cuernos \\ para introducir el miedo en el corazón \\ de los habitantes de Jericó". \\ Teniente coronel Dan Kuehl, \\ retirado del servicio activo, experto \\ en operaciones especiales psicológicas ${ }^{1}$.
}

“iEs un pecado! iEs un pecado! iEs un pecado! (...) iUsar a Ludwig Van así! iBeethoven solo compuso música!”.

Alex DeLarge en La Naranja Mecánica.

\section{Introducción}

No es un secreto la utilización de música con fines ideológicos por parte de Estados Unidos. La política de seguridad del país de las franjas y las estrellas tomó esfuerzos redoblados a raíz de los atentados del 11-S, entre éstos la apertura de bases ilegales externas para el tratamiento de detenidos acusados de terrorismo. A partir de la filtración de reportes de prensa que evidenciaron prácticas de tortura en estos centros, incluido el uso de ciertas canciones bajo los denominados no-touch methods; algunos músicos, amparados en la Ley de Libertad de Información, exigieron desclasificar los informes concernientes.

En 2008, en un trabajo de investigación simultáneo, la revista americana de izquierda Mother Jones y el periódico The Guardian publicaron una lista de canciones y un reportaje basado en entrevistas a soldados y expresidiaros de la bahía de Guantánamo, lo cual dio paso a polémicas declaraciones, tanto del gobierno como de las agrupaciones y músicos involucrados, quienes no estaban enterados sobre el uso de sus obras.

Este artículo analiza de forma resumida los aspectos centrales que han sido objeto de debate alrededor del tema. Inicia con un acercamiento a los orígenes del uso de la música como tortura y aborda la distinción música-sonido en su práctica, así como a los escenarios en los que se lleva a cabo.

Más adelante se hace una revisión mediática de los acontecimientos, desde los primeros reportes en 2003 hasta el año en curso, presentando las opiniones encontradas de algunos músicos involucrados. Se

1 Fue la respuesta que el exmilitar dio en 2004 al St. Petersburg Times (ahora Tampa Bay Times), cuando se le cuestionó sobre el uso de la música como técnica de tortura. 
discute el fenómeno de ciertas deformaciones discursivas, en relación con las temáticas y mensajes de algunas canciones, y se cierra con un breve apartado sobre la preferencia observada hacia un género musical específico.

\section{Antecedentes}

El uso de la música como tortura no es algo nuevo. Puede rastrearse desde la Alemania nazi ${ }^{2}$, y en una etapa de experimentación posguerra bajo programas de investigación financiados por la CIA y por otras agencias de países aliados ${ }^{3}$.

"Desde sus inicios, los comandantes de los campos de concentración nazi hicieron uso deliberado de la música para quebrar a los prisioneros mentalmente, y para robarles su dignidad e identidad cultural. También la utilizaban para lograr fines ideológicos"4. Así lo explica el musicólogo de la Universidad de Freiburg en Alemania, Guido Flacker, en un estudio sobre el uso de la música transmitida por el sistema de altavoces en el campo de concentración de Dachaur, un centro de primera generación, pionero en incorporar tecnología moderna a las actividades de adoctrinamiento en 1933. Entre otras funciones, de acuerdo con el autor, tuvo la de educar a los prisioneros bajo principios nacionalistas, con el entendido de mostrar la superioridad racial y cultural alemana, por lo que podía escucharse desde música de marcha hasta el vals Blue Danube de Strauss, o Preislied de la ópera Meistersinger de Wagner. También canciones como Deutschland erwache aus deinem schweren Traum! [iAlemania despierta de su pesadilla!] y Brüder, zur Sonne, zur Freiheit! [iHermanos, hacia el sol, hacia la libertad!], además de los discursos oficiales del partido fascista ${ }^{5}$.

En particular para los prisioneros políticos, las transmisiones de radio de Dachau implicaban una forma de discriminación y de terror mental. Constantemente se les hacía tomar conciencia del hecho de que estaban en manos de sus enemigos y sin ningún medio para defenderse. Wenzel Rubner escribió: "Para nosotros, la radio no representaba un entretenimiento, sino una nueva forma de tormento para nuestras almas. Debíamos escuchar los discursos del Führer y sus brutales insultos hacia nosotros y nuestros camaradas. Nos obligaban a oír las canciones que se burlaban de nuestras creencias"6.

2 G. Fackler, 2002.

3 A. McCoy, A Question of Torture: CIA Interrogation, from the Cold War to the War on Terror.

4 G. Fackler, op. cit.

5 Idem.

6 Idem. 
Basado en otros testimonios, Flacker menciona que además de disimular los gritos de los presos durante los interrogatorios, la música en alto volumen desinhibía a los torturadores, induciéndolos a un estado eufórico que les distraía de sus acciones.

Casi 20 años más tarde, en el transcurso de las décadas de los 50’s y 60's, mientras Estados Unidos estaba en plena labor propagandística de la mano de jazzistas como Louis Armstrong y Dizzy Gillespie, con la campaña de los "buenos embajadores", la CIA financiaba junto a los servicios de inteligencia de Canadá y Gran Bretaña, un programa de investigación en torno a estudios sobre el dolor auto-inducido, en las universidades de Cornell, Yale y McGill. A estas primeras experimentaciones se incorporarían más tarde las posiciones de estrés y humillaciones personales, sexuales o culturales, así como la privación sensorial y la exposición a ruido continuo en distinto volumen; incluso, se analizó la capacidad de personas comunes para causar dolor a otros hasta la muerte ${ }^{8}$.

En 1989, las tropas estadounidenses hostigaron durante días al entonces presidente de Panamá Manuel Noriega, bombardeándolo con música mientras permanecía acuartelado en la embajada del Vaticano, seguido de una rendición paulatina. Los soldados usaron un casette de $\mathrm{AC} / \mathrm{DC}$.

La anécdota fue narrada como un hecho incidental por Ben Abel, vocero de la armada de Fort Bragg, en 2004, para el St. Petesburg Times, y mencionó que desde esa ocasión, y tras dar cuenta de su efectividad, la milicia usó con más frecuencia los altavoces, interesados especialmente en equipos portátiles para cada soldado, aunque esta declaración se confronta con un segmento del documental Songs of War (2012), en el que se evidencia el uso de altavoces móviles a modo de mochilas, o en equipos similares a generadores pequeños de corriente desde la guerra de Vietnam; así como el uso de líneas de altavoces ajustadas a los helicópteros, algo que recuerda a algunas escenas de la película de 1979 Apocalypse Now, de Francis Ford Coppola; sin embargo, no reproducían precisamente las Valkirias de Wagner.

En el mismo documental, un veterano de guerra norteamericano narra las acciones de la campaña llamada "El alma errante", nombrada así a partir de una tradición vietnamita, en la cual, entre más lejos moría un soldado de su hogar, su alma se condenaba a vagar de manera indefinida. Los sonidos que reproducían en las líneas de audio solían ser canciones populares fúnebres, en cuyo fondo se escuchaba la voz de un hombre hipotéticamente muerto, algún padre de familia anunciando a

7 D. Carletta, “Those white guys are working for me’: Dizzy Gillespie, Jazz, and the cultural politics of the Cold War during the Eisenhower Administration", International Social Science Review. 
sus seres queridos sobre su inevitable partida. La intención era desmoralizar al enemigo.

Durante la guerra contra Irak, el ejemplo representativo fue el de la toma de Fallujah, en noviembre de 2004, donde los sistemas LRAD ${ }^{9}$, incorporados a las unidades de operación psicológica (PsyOps), entonaron Hell's Bells y Shoot to Thrill de AC/DC, así como Enter Sandman de Metallica. Ben Abel dijo que la armada no tenía una lista oficial de canciones para usar en combate, y agregó:

\begin{abstract}
... nuestros hombres han demostrado una gran creatividad a la hora de escoger sonidos que creen que afectarían al enemigo [...] ellos tienen sus propios reproductores de mini-disc con su propia música, además de cientos de sonidos bajados de Internet. Escogen las canciones de acuerdo al gusto personal. Tenemos a gente muy joven tomando estas decisiones" ${ }^{\prime 10}$.
\end{abstract}

\title{
¿Música o sonido?
}

Las posturas más generales sobre la efectividad de la música como tortura son las que hacen referencia a su propiedad física en cuanto a sonido, y cuya eficacia depende de una exposición prolongada en alto volumen. En palabras de Ben Abel: "No es tanto la música como el sonido, es como lanzar una bomba de humo. El objetivo es desorientar y confundir al enemigo para ganar ventaja táctica"11. Por su parte, el jefe del Departamento de Psicología Clínica para la Academia de Ciencias Médicas Rusa, Serguéi Yenikólopov, dijo en el sitio web de la radio La Voz de Rusia:

\footnotetext{
Nuestro oído está estructurado de tal forma que las diversas frecuencias influyen en él de diferente manera. Hay frecuencias que producen una gran molestia. En segundo lugar, el sonido fuerte, el alto nivel de los decibelios es, en primer lugar, un estrés para el oído y también para el cerebro. Y, en tercer lugar, el ritmo de la música puede ser asimismo insoportable. Estos tres métodos de influencia sobre el cerebro humano y, a través de él en la psique, se utilizan con frecuencia durante las torturas ${ }^{12}$.
}

Por su parte, la profesora de música en la Universidad de Artes y Ciencias de Nueva York, Suzanne G. Cusick, comentó para The Washington Post: "El sonido, a un nivel muy alto, crea una saturación sensorial que rompe la subjetividad y puede llegar a una regresión y un comportamiento infantil [...] su efectividad depende de la constancia del sonido, no de la calidad de la música ${ }^{13}$.

9 Aparato Acústico de Largo Alcance, o LRAD (Long Range Acoustic Device). Puede observarse un modelo en funcionamiento en el siguiente link: https://www.youtube.com/watch?v=iSvrqEAT_k0

10 L. DeGregory, 2004.

11 Idem.

12 A. Kóbzev, 2013.

13 J. Heim, 2009. 
Si bien Cusick remarca esta postura, abre paso a otras consideraciones al tomar en cuenta la función del daño en los dos escenarios hasta ahora vistos:

\begin{abstract}
La premisa en común es que el sonido puede lastimar seres humanos, sin matarnos, en una amplia variedad de maneras. Lo que diferencia el uso del sonido o de la música en el campo de batalla y el uso del sonido o de la música en los cuartos de interrogatorios, es la ubicación declarada del daño. Los teóricos de su uso en el campo de batalla enfatizan el efecto del sonido en el cuerpo, mientras que los teóricos del cuarto de interrogatorios se enfocan en la capacidad que tienen la música y el sonido para destruir la subjetividad. Aquí hay algo sobre la intersección de la relación entre mente y cuerpo con la distinción entre espacio privado y público, y la jerarquía de comandos y operaciones de campo, que en un futuro quisiera discutir más a fondo ${ }^{14}$.
\end{abstract}

No obstante aunque resulta obvia la aplicación de la música en cuanto su propiedad física como sonido, los gustos en la selección musical por parte de los soldados no son una simple coincidencia como se verá más adelante, sino un fenómeno que termina involucrando aspectos discursivo-culturales más diversos y complejos.

\title{
Primeros reportes
}

En abril de 2004, The New Yorker destapa la historia sobre las prácticas de tortura en la base de Abu Ghraib, en Irak ${ }^{15}$, una de las prisiones ilegales de Estados Unidos para cautivos de guerra. La cadena americana CBS mostraría más adelante, en su programa 60 Minutes, las conocidas fotografías ilustrando los abusos cometidos. No obstante, un año antes, en mayo de 2003, la británica BBC ya había publicado un reportaje que revelaba de forma concreta el uso de música como tortura, dando los primeros nombres de canciones que sonaban en los oídos de los presos durante sesiones previas a los interrogatorios: el tema Plaza Sésamo del programa de televisión homónimo, el tema de Barney, el dinosaurio morado, y Enter Sandman de Metallica.

Esto fue el inicio de una serie de "destapes" continuos durante los cuatro años posteriores, en los que siguieron saliendo a la luz pública tanto material fotográfico sobre abusos físicos como de "tortura sin contacto", con más temas musicales mencionados por ex presidiarios que testimoniaron para diversos medios. Los reportes sorprenderían a los músicos, quienes lo tomaron al principio como una broma. Destaca el caso del compositor del tema Plaza Sésamo, Christopher Cerf, quien

14 S. Cusick, 2006, pp. 8-9.

15 S. Hersh, 2004. 
participaría más tarde como conductor en el documental Songs of War (2012), producido por la cadena Al Jazeera.

En 2008, dos años después del cierre de la base de Abu Ghraib, la periodista Justine Sharrock publicó en la revista Mother Jones una lista de canciones obtenida a través de entrevistas anónimas con soldados de la bahía de Guantánamo y exprisioneros. El "playlist” fue un fenómeno en la red y aún se encuentra circulando en múltiples blogs y páginas que abordan el tema. La lista es la siguiente ${ }^{16}$ :

01. Deicide - Fuck Your God

02. Dope - Die Motherfucker Die

03. Dope - Take Your Best Shot

04. Eminem - White America

05. Eminem - Kim

06. Barney - Barney Theme Song

07. Drowning Pool - Bodies

o8. Metallica - Enter Sandman

09. Anuncio de TV - Meaw Mix

10. Series de TV - Plaza Sésamo

11. David Gray - Babylon

12. Bruce Springsteen - Born in the USA

13. AC/DC - Shoot to Thrill

14. AC/DC - Hell's Bells

15. Bee Gees - Stayin' Alive

16. Tupac Shakur - All eyes on me

17. Christina Aguilera ft. Redman - Dirty

18. Neil Diamond - America

19. Rage Against The Machine - Bulls on Parade

20. Don McClean - American Pie

21. Saliva - Click Click Boom

22. Matchbox 20 - Cold

23. Hed P. E. - Swan Dive

24. Prince - Raspberry Beret

Otras canciones mencionadas en testimonios y en reportes posteriores son: Sweet Dreams, versión de Marilyn Manson, Baby One More Time de Britney Spears, Don't Gimme no Lip de Pearl Jam, Somewhat Damaged de Nine Inch Nails y Killing in the Name de Rage Agains the Machine. Otros grupos mencionados (sin saber específicamente qué canciones) son R.E.M., James Taylor, Red Hot Chilli Peppers y el rapero Doctor Dre.

16 J. Sharrock, 2008a. 
Se anexa recientemente la banda Skinny Puppy, al levantar una demanda por 666 ooo dólares luego de enterarse sobre el uso de su música en Guantánamo, según una nota de Infowars de febrero de 2014.

La lista de Sharrock sería seguida por el reportaje del abogado Clive Stafford, publicado en The Guardian en junio de 2008. Tras la información divulgada, algunos músicos se ampararon en la Ley de Libertad de Información para que la administración de Estados Unidos desclasificara todos los informes relacionados con el tema. Meses después se organizaba un movimiento en apoyo al cierre de la base de Guantánamo, un compromiso pendiente que el presidente Barack Obama dijo que realizaría para el 22 de enero de $2010^{17}$.

Según Stafford, The Guardian intentó contactar con todos los músicos y agrupaciones, pero la mayoría decidió no emitir comentarios al respecto, mientras otros, como David Gray, respondieron al llamado. Haj Ali, el hombre en la famosa fotografía del prisionero encapuchado, sujeto a cables de corriente por manos y genitales, habló sobre la privación del sueño que sufrió con la canción Babylon del mismo artista. Gray menciona al respecto:

\begin{abstract}
Es sorprendente que no haya más de una protesta. Yo firmo con mucho gusto una petición para no usar mi música, pero parece que haría falta hacer un poco más [...] Las sutilezas morales de si están usando mi canción o no son totalmente irrelevantes. Estamos pensando por debajo del nivel de la gente a la que se supone nos oponemos, y va en contra de toda nuestra historia y todo lo que decimos representar, es en verdad repugnante ${ }^{18}$.
\end{abstract}

James Hetfield, vocalista de Metallica, ha hecho diversas declaraciones. En una de ellas, comentó bromeando: "Hemos estado castigando a nuestros padres, nuestras mujeres, y seres queridos con nuestra música desde siempre. ¿Por qué tendría que ser distinto con los iraquíes?" En una posterior entrevista para el canal alemán 3Sat, comentaría:

\footnotetext{
Parte de mí está orgullosa porque hayan escogido a Metallica [...] Es fuerte, es música poderosa. Representa algo que a ellos quizás no les gusta... quizás libertad, agresión... no lo sé... libertad de expresarse [...] Luego otra parte de mí se preocupa por la gente que nos trata de ligar con algún movimiento político producto de eso. Nosotros no tenemos nada que ver ahí, y tratamos de hecho de ser lo más apolíticos posible. Pienso que política y música, al menos para nosotros, no se ligan. La política separa a la gente y nosotros la queremos unida. No puedo decirles "háganlo" ni tampoco "no lo hagan" ${ }^{20}$.
}

Steve Asheim, baterista de Deicide, la banda de death metal que encabe-

17 AP, 2008; I. Piquer, 2009; H. Areyuna, 2014.

18 C. Stafford, 2008.

19 Idem.

20 3Sat, 2013. 
za la lista de Sharrock con la canción Fuck Your God, dijo sentirse a gusto sabiendo que el trabajo del grupo contribuía a las tropas, y agregó:

\begin{abstract}
Esos tipos no son un montón de chiquillos de secundaria. Son guerreros y están entrenados para resistir la tortura. Ellos esperan ser quemados con antorchas o ser golpeados hasta quebrar sus huesos. Si yo fuera prisionero en Guantánamo y me bombardearan con música, sería como: ceso es todo lo que tienen?, ipor favor! Desde luego no creo en torturar a la gente, pero tampoco creo que la música a alto volumen sea una tortura [...] No creo que haya una sala donde ellos escojan canciones para molestar a los prisioneros ${ }^{21}$.
\end{abstract}

Trent Reznor, de Nine Inch Nails, mencionó que no había algo más ofensivo, insultante y degradante que saber que la música en la que ha puesto esfuerzo y corazón sea usada como tortura ${ }^{22}$. Por el contrario, en Songs of War (2012), el bajista de la banda Drowning Pool opinó sobre el uso de su canción Bodies: "Algunos asumen que nos sentimos ofendidos porque los militares piensen que nuestra canción es perfecta para derrumbar a alguien psicológicamente. Pero creo que es un honor que quizás con nuestra canción se evite otro ataque como el 11-S o alguno similar".

\title{
Deformaciones discursivas
}

En contraposición a la postura de Asheim sobre el perfil de los presos, están los casos de presidiarios que no son culpables. El más conocido, el proceso de Moazzam Beg, quien después de los atentados del 11-S se encontraba como ciudadano británico en Pakistán a lado de su familia, donde fue detenido con violencia y trasladado a Guantánamo. Pasó tres años sujeto a las prácticas de tortura de la base, hasta que fue puesto en libertad en 2005.

Moazzam explica en Songs of War (2012), que muchas de las canciones a las que fue expuesto en alto volumen pertenecían a bandas que eran conocidas para él y que incluso solía escuchar, como Marilyn Manson o Metallica. Esto aminoraba la función "desmoralizante" de la tortura al poder distinguir y reconocer los audios, aunque se lamentaba por los presos que no podían hacer esa diferencia musical; por lo tanto, al no estar relacionados con esos sonidos “occidentales”, se vencían más fácil ante los efectos de la tortura sonora en adición a los castigos físicos; lo cual a la larga podía inducir al preso a un estado casi vegetativo, desconcertado y listo para asentir cualquier declaración que se le pidiera durante los interrogatorios.

Por otro lado, basta con dar un vistazo a los títulos de las canciones

21 C. Stafford, 2008.

22 A. Salazar, 2014. 
en la lista de Sharrock y anexas. En realidad son sugerentes si se piensa además, que se están empleando en su mayoría a prisioneros de Oriente Medio mientras se encuentran en posición de estrés: encadenados al suelo, inmovilizados, encapuchados y expuestos a bajas temperaturas. "A la mierda tu dios", "Muere hijo de puta muere", "Todos los ojos sobre mí", "Una vez más bebé", "Dulces sueños", incluso la anodina Barney con su estribillo "te quiero yo y tú a mí" o "Permaneciendo vivo" de los Bee Gees parecen insinuaciones ad hoc. Por otro lado, títulos como "América", "América Blanca", "Nacido en U.S.A." o "Pastel Americano", parecieran apelar a cierto espíritu nacionalista.

A pesar de lo anterior, muchas de las canciones contienen un discurso completamente distinto, incluso antisistema. El ejemplo que salta es Born in the U.S.A. de Bruce Springsteen, que repite escenario luego de que fuera apropiada como himno de campaña por el presidente Donald Reagan en 1984, mientras este buscaba la reelección. La letra es una crítica a los atropellos del gobierno frente a los soldados y los caídos durante la Guerra de Vietnam, aunque a Reagan le pareció patriótica.

Marcyliena Morgan, directora del archivo de Hip-Hop en el departamento de Estudios Americanos y Afroamericanos en la Universidad de Harvard, comentó para el diario La Vanguardia: "Es irónico, porque muchas canciones de Eminem y Doctor Dre instan a las víctimas de injusticias sociales a rebelarse" ${ }^{23}$. Otro ejemplo es la banda contestataria Rage Against the Machine, que siempre se ha mostrado en contra de estas prácticas. El guitarrista Tom Morello expresó: "El hecho de que la música que ayudé a crear haya sido utilizada en crímenes contra la humanidad me enferma" 24 .

Todo indica que las canciones sí son seleccionadas, aunque eso no garantiza la comprensión de su discurso. Esto hace pensar en que los soldados podrían hacer la selección solo a partir del título sin importarles el significado de la letra (es decir sacándolas de contexto), o que las letras (al menos para aquellas que tienen un "doble mensaje") no son entendibles para ellos. Una tercera opción: quizá lo sepan pero lo pasan por alto. El propio Asheim menciona irónicamente: "La mayoría de las personas que escuchan este tipo de música, no dan una mierda sobre un mensaje político. Ellos solo quieren Rock"25.

El reporte de la BBC en 2003 señaló que los prisioneros habían sido expuestos a música "culturalmente ofensiva", o en sus propias palabras, "insoportablemente dura", "occidental” e "infiel”. Justine Sharrock refiere:

23 A. Robinson, 2005.

24 J. Heim, 2009.

25 C. Stafford, 2008. 
Algunas canciones elegidas por los soldados como Die Motherfucker Die y Bodies (con letras como "deja que los cuerpos golpeen el suelo"), no sólo asustaban a los detenidos, sino que aumentaban el sentimiento bélico y el deseo de matar de los soldados. Otras, como Born in the USA o America, himnos del patriotismo estadounidense, no sólo reafirmaban la determinación de los soldados, sino que también servían para hacer alarde ante los detenidos de la superioridad esta-

dounidense ${ }^{26}$.

"Se dice que grabaciones de Britney Spears y Cristina Aguilera han sido utilizadas contra detenidos específicos: quien recibió el tratamiento Aguilera era un angloparlante, se puede asumir por ello que las letras sexualmente provocativas eran parte de la intención" ${ }^{27}$. Las observaciones anteriores recuerdan a las mismas funciones de la música en los campos de concentración nazi: denigraciones culturales, imposiciones nacionalistas y estímulo o catarsis para los soldados; un extra a la propiedad física de la música en cuanto sonido.

\section{La predilección por un género}

La lista de Sharrock es, sin duda versátil; sin embargo, hay un género predominante, tanto en los interrogatorios como en el frente de batalla. En 2008, el mismo año en que Mother Jones publicara la peculiar lista, el antropólogo canadiense Sam Dunn lanzó el documental Global Metal, segunda parte de su primer trabajo Metal: A Headbanger's Journey de 2005. La producción se centró en la escena del metal en países que se encuentran en medio de conflictos bélicos o que pasaron por dictaduras, como Brasil, Indonesia, China e Irán.

En esta producción, la música metal se muestra como una válvula de escape y de expresión cultural para las nuevas generaciones, quienes incorporan nuevos ideales y estéticas al movimiento según el lugar de origen. Cuatro años antes, el párrafo de inicio en la nota del St. Petesburg Times le dio al término "válvula de escape" otras connotaciones:

Mientras los tanques se preparaban para abatir Fallujah, y las tropas estadou-
nidenses empezaban a rodear la ciudad, los oficiales de operaciones especiales
revolvieron sus cajas de CD's en busca de una pista de audio para estimular el
asalto. ¿Qué podría irritar más a los insurgentes: ladridos de perros o bluegrass,
bebés gritando o un chillante feedback? Heavy Metal. El arma letal del ejército ${ }^{28}$.

En Songs of War (2012), varios de los soldados en campo expresan su gusto especial por el metal al resultarles música "motivante". Uno de ellos 26 J. Sharrock, 2008b.

27 S. Cusick, 2006, p. 14.

28 L. DeGregory, 2004. 
menciona como preferencias personales las canciones Reign in Blood y Angel of Death de la banda de thrash metal Slayer. La segunda canción ha sido objeto de crítica desde su publicación en 1986, tildando a la agrupación de racista o neonazi debido a la temática, en la que se detallan los experimentos del médico Josef Mengele en el campo de Auschwitz. La banda ha negado siempre las acusaciones y parece entender su obra simplemente como un producto comercial más dentro de la industria de la música. En Metal: A Headbanger's Journey (2005), el vocalista y bajista Tom Araya respondería sobre el nombre de su álbum de 2001 God Hate Us All: "Dios no nos odia a todos... pero es un gran título".

Araya esperaba una reacción similar a la causada por Angel of Death con la canción Jihad, incluida en el álbum Christ Ilussion de 2006, en la que se narran los atentados del 11 de septiembre desde la perspectiva de un terrorista, pero ésta pasó desapercibida ${ }^{29}$.

En 2013, la revista Esquire publica una entrevista con uno de los miembros del equipo SEAL, que se encargó de la muerte de Osama Bin Laden. El militar bajo el pseudónimo “The Shooter” reveló:

Cuando comenzó la guerra en Irak, se utilizó la música de Metallica para ablandar a la gente antes de que fueran interrogados. La banda se enteró de esto y nos dijeron: "Oye, por favor no utilices nuestra música, porque no queremos promover la violencia”. Pensé: Amigo, tienes un álbum llamado Kill’ Em All.

Sin embargo, dejamos de usar su música, y luego una banda llamada Demon Hunter se puso en contacto con nosotros y nos dijeron: "Todos estamos de acuerdo con lo que hacen". Nos enviaron CD's y parches. Me puse mi parche de Demon Hunter en cada misión. Me lo puse cuando ataqué a Bin Laden ${ }^{30}$.

Cusick ofrece otra hipótesis sobre la inclinación que tienen los soldados a la música metal como apropiada en los escenarios de batalla:

\footnotetext{
El heavy metal y el rap, géneros generalmente codificados como masculinos dentro de la cultura popular estadounidense, son escuchados, para quienes no se identifican con ellos, como música que incorpora los sonidos de la ira masculina. Por eso puede parecerle, a los soldados en el terreno, que esa música "tortura" a los hombres musulmanes creando un espacio sonoro en el que los hombres de Estados Unidos los vencen en una lucha de masculinidades. Algunas de las canciones específicas usadas en batalla (Enter Sandman de Metallica, Hell's Bells de AC/DC) parecen tener textos apropiados para preparar a ambos lados para la confrontación con la muerte macabra, que muchos recuentos o memorias de militares asemejan al éxtasis ${ }^{31}$.
}

29 L. Lahtinen, 2007.

30 P. Bronstein, 2013.

31 S. Cusick, 2006, p. 14. 


\title{
Conclusiones
}

En la guerra global contra el terrorismo, la industria musical no queda desestimada entre los arsenales militares destinados a la tortura. La selección de canciones hecha por los soldados presenta similitudes tanto en el frente de batalla como en los escenarios de interrogatorio, donde la música metal ocupa hasta ahora los lugares preferenciales.

Los motivos que tuvieron en su momento los campos de concentración nazis, respecto al uso de la música, no difieren de los criterios de la "tortura sin contacto" americana. Como bien menciona Cusick: "La música, entonces, no es únicamente un componente de la 'tortura sin contacto' sino también un componente de la pretensión simbólica de soberanía global por parte de los Estados Unidos" ${ }^{2}$.

Es importante el énfasis de las circunstancias globales en la guerra y en la facilidad con la que las nuevas tecnologías pueden incorporar determinadas prácticas, que a la larga pueden derivar en fenómenos disímiles según el terreno en que son abordados. El caso de los discursos, la temática en las letras, y las deformaciones cultural-contextuales, puede analizarse también, tomando en cuenta lo dicho en su momento por el teniente coronel Dan Kuehl:

\begin{abstract}
Con la creciente globalización del mundo, sabemos que algunos iraquíes escuchan música americana, incluso metal, en el Internet, la radio y la televisión. Aun durante el apogeo de los talibanes, podían obtener videos y música occidental. Aunque algunos insurgentes pudieron estar temblando horrorizados por el bombardeo de Metallica, o abandonando sus puestos frente al ruido espantoso, otros podrían haber estado alzando los puños al ritmo de los riffs familiares, sintiéndose igualmente estimulados que los americanos ${ }^{33}$.
\end{abstract}

Tal como la música y la guerra, el debate sigue en pie.

\section{Referencias}

AL JAZEERA (2012). Songs of War. Catar. Video Documental. [Recuperado el 11 de abril de 2014 de: http://www.aljazeera.com/programmes/aljazeeraworld/2012/05/201253072152430549.html]

AREYUNA, H. (enero 30, 2014). Cierre de la cárcel de Guantánamo: la eterna promesa de Barack Obama. Diario Uchile. [Recuperado el 11 de abril de 2014 de: http://radio.uchile.cl/2014/o1/30/cierre-de-lacarcel-de-guantanamo-la-eterna-promesa-de-barack-obama]

32 Ibid., p. 16.

33 L. DeGregory, 2004. 
AP (septiembre 12, 2008). Musicians don't want tunes used for torture. Today. [Recuperado el 11 de abril de 2014 de: http://www.today.com/id/28144557/ns/today-today_entertainment/t/musicians-dont-want-tunes-used-torture/\#.Uo95c-Z5M9A]

BBC (mayo 20, 2003). Sesame Street breaks Iraqi POWs. [Recuperado el 11 de abril de 2014 de: http://news.bbc.co.uk/2/hi/middle_ east/3042907.stm]

BRONSTEIN, P. (febrero 11, 2013). The man who killed Osama Bin Laden... is screwed. Esquire Magazine. [Recuperado el 11 de abril de 2014 de: http://www.esquire.com/features/man-who-shot-osamabin-laden-0313]

CARLETTA, D. (2007). “Those white guys are working for me’: Dizzy Gillespie, Jazz, and the cultural politics of the Cold War during the Eisenhower Administration”, International Social Science Review. 3, 4 (82) pp. 115-134.

CUSICK, S. (2006). La música como tortura / La música como arma. TRANS-Revista Transcultural de música 10. Traducción de Sebastián Cruz y Ruben Lopez Cano. [Recuperado el 10 de abril de 2014 de: http://www.sibetrans.com/trans/articulo/153/la-musica-como-tortura-la-musica-como-arma]

DEGREGORY, L. (noviembre 21, 2004). Iraq 'n' roll. St Petersburg Times. [Recuperado el 14 de abril de 2014 de: http://www.sptimes. com/2004/11/21/news_pf/Floridian/Iraq_n__roll.shtml]

DUNN S. y S. McFayden (2005). Metal: A Headbanger's Journey. Canadá. Video Documental. Colección personal.

. (2008). Global Metal. Canadá. Video Documental. Colección Personal.

FACKLER, G. (2002). Tortura sónica en Dachaur. La Música y el Holocausto. [Recuperado el 10 de abril de 2014 de: http://holocaustmusic.ort.org/es/places/camps/music-early-camps/dachau/sonic-torture-dachau/]

HEIM, J. (octubre 22, 2009). Musicians seek Guantanamo records on detainee torture. The Washington Post. [Recuperado el 13 de abril de 2014 de: http://www.washingtonpost.com/wp-dyn/content/article/2009/10/21/AR2009102103743.html]

HERSH, S. (mayo 10, 2004). Torture at Abu Ghraib. The New Yorker. [Recuperado el 11 de abril de 2014 de: http://www.newyorker.com/ archive/2004/05/10/040510fa_fact?currentPage=all]

KÓBZEV, A. (septiembre 17, 2013). Con las canciones de Iglesias torturaban a los enemigos de Pinochet. La voz de Rusia. [Recuperado el 16 de abril de 2014 de: http://spanish.ruvr.ru/2013_09_17/ 
Con-las-canciones-de-Julio-Iglesias-torturaban-a-los-enemigos-de-Pinochet-5874/]

LAHTINEN, L. (diciembre 17, 2007). Jeff Hanneman Interview. Metal Rules. [Recuperado el 15 de abril de 2014 de: http://www.metal-rules.com/zine/index.php?option=content\&task=view\&id=737\&Ite$\operatorname{mid}=60]$

MCCOY, A. A Question of Torture: CIA Interrogation, from the Cold War to the War on Terror. Metropolitan, Nueva York, 2006.

PIQUER, I. (octubre 23, 2009). No con mi música. Público.es. [Recuperado el 11 de abril de 2014 de: http://www.publico.es/culturas/263162/ no-con-mi-musica]

ROBINSON, A. (diciembre 22, 2005). Tortura a ritmo de rap y heavy. La Vanguardia. [Recuperado el 16 de abril de 2014 de: http://www. metallitarium.com/files/pdf/LVG200512220371LB.pdf]

SALAZAR, A. (febrero 6, 2014). Industrial Band Skinny Puppy Bills DoJ For Using Its Music as a Torture Device. Infowars.com. [Recuperado el 12 de abril de 2014 de: http://www.infowars.com/industrialband-bills-doj-for-using-its-music-as-a-torture-device/]

SCOTT, J. C. Los dominados y el arte de la resistencia. Era, México, 2000.

SHARROCK, J. (febrero 22, 2008a). The Torture Playlist. Mother Jones Magazine. [Recuperado el 10 de abril de 2014 de: http://www.motherjones.com/politics/2008/02/torture-playlist]

(2008b). Enloquecer a ritmo de heavy. Asociación de Estudiantes y Graduados Universitarios Adventistas de España. Documento de Convención. [Recuperado el 14 de abril de 2014 de: http://www. aeguae.org/convencion/Musica_para_la_tortura.pdf]

STAFFORD, C. (junio 19, 2008). Welcome to 'the disco'. The Guardian. [Recuperado el 11 de abril de 2014 de: http://www.theguardian.com/ world/2008/jun/19/usa.guantanamo]

3Sat. (2013). Entrevista con James Hetfield Metallica Full 3Sat. Alemania. Video. [Recuperado el 14 de abril de 2014 de: https://www.youtube.com/watch?v=iEtL57_nqv4] 